\title{
The nature of the 2014-2015 dim state of RW Aurigae revealed by X-ray, optical, and near-IR observations ${ }^{\star}$
}

\author{
P. C. Schneider ${ }^{1}$, H. M. Günther ${ }^{2}$, J. Robrade ${ }^{3}$, S. Facchini ${ }^{4}$, K. W. Hodapp ${ }^{5}$, C. F Manara ${ }^{1}$, V. Perdelwitz ${ }^{3}$, \\ J. H. M. M. Schmitt ${ }^{3}$, S. Skinner ${ }^{6}$, and S. J. Wolk ${ }^{7}$ \\ ${ }^{1}$ European Space Research and Technology Centre (ESA/ESTEC), Keplerlaan 1, 2201 AZ Noordwijk, The Netherlands \\ e-mail: cschneider@hs.uni-hamburg.de \\ 2 Massachusetts Institute of Technology, Kavli Institute for Astrophysics \& Space Research, 77 Massachusetts Avenue, Cambridge, \\ MA 02139, USA \\ 3 Hamburger Sternwarte, Gojenbergsweg 112, 21029 Hamburg, Germany \\ 4 Institute of Astronomy, University of Cambridge, Madingley Road, Cambridge, CB3 0HA, UK \\ 5 Institute for Astronomy, 640 N. A'ohoku Place, Hilo, HI 96720, USA \\ ${ }^{6}$ University of Colorado, 392 UCB, Boulder, CO 80309, USA \\ 7 Harvard-Smithsonian Center for Astrophysics, 60 Garden Street, Cambridge, MA 02139, USA
}

Received 24 August 2015 / Accepted 27 October 2015

\section{ABSTRACT}

\begin{abstract}
The binary system RW Aur consists of two classical T Tauri stars (CTTSs). The primary recently underwent its second observed major dimming event ( $\Delta V \sim 2 \mathrm{mag}$ ). We present new, resolved Chandra X-ray and UKIRT near-IR (NIR) data as well as unresolved optical photometry obtained in the dim state to study the gas and dust content of the absorber causing the dimming. The X-ray data show that the absorbing column density increased from $N_{\mathrm{H}}<0.1 \times 10^{22} \mathrm{~cm}^{-2}$ during the bright state to $\approx 2 \times 10^{22} \mathrm{~cm}^{-2}$ in the dim state. The brightness ratio between dim and bright state at optical to NIR wavelengths shows only a moderate wavelength dependence and the NIR color-color diagram suggests no substantial reddening. Taken together, this indicates gray absorption by large grains $(\gtrsim 1 \mu \mathrm{m})$ with a dust mass column density of $\gtrsim 2 \times 10^{-4} \mathrm{~g} \mathrm{~cm}^{-2}$. Comparison with $N_{\mathrm{H}}$ shows that an absorber responsible for the optical/NIR dimming and the X-ray absorption is compatible with the ISM's gas-to-dust ratio, i.e., that grains grow in the disk surface layers without largely altering the gas-to-dust ratio. Lastly, we discuss a scenario in which a common mechanism can explain the long-lasting dimming in RW Aur and recently in AA Tau.
\end{abstract}

Key words. stars: individual: RW Aur - stars: pre-main sequence - stars: low-mass - stars: variables: T Tauri, Herbig Ae/Be X-rays: stars

\section{Introduction}

Classical T Tauri stars (CTTSs) are young stars accreting from their surrounding protoplanetary disk. The disk's gas-to-dust ratio is challenging to measure directly. Therefore, the interstellar medium (ISM) ratio of 100:1 is regularly assumed (see review by Williams \& Cieza 2011). In the standard magnetospheric accretion model, matter is channeled along magnetic field lines from the inner edge of the disk onto the stellar surface (Bouvier et al. 2007).

The young binary RW Aurigae (distance $140 \mathrm{pc}$ ) is among the well-studied CTTS with dense photometric and spectroscopic monitoring. It is composed of a jet-driving primary and a secondary separated by 1 .' 4 ; the masses of both stars are thought to be approximately solar. The system ( $\mathrm{A}+\mathrm{B}$ combined) shows complex periodic optical variability. Its magnitude and color change by a few tenths of mag on time scales of 2-5 days (e.g., Petrov et al. 2001a,b). Additional irregular variability of up to two magnitudes along the reddening vector is superimposed onto this periodic variability (e.g., Grankin et al. 2007; Petrov \& Kozack 2007). Observations of CO emission show that the primary's disk is truncated at $R<60 \mathrm{AU}$ and that diffuse emission extends well beyond the disk; both features are best explained by a close ( $\sim 70 \mathrm{AU})$ approach of RW Aur B around A

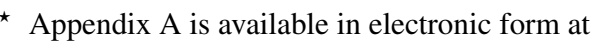
http://www. aanda.org
}

(Cabrit et al. 2006; Dai et al. 2015). A half-year dimming event in 2011 ( $\Delta V \approx 2 \mathrm{mag}$ ) was attributed to a "bridge" of material related to the extended $\mathrm{CO}$ structure connecting both components (Rodriguez et al. 2013; Dai et al. 2015).

Towards the end of 2014, the system dimmed again by 2 mag. The similarity of both dimming events could suggest that the "extra absorber" in 2014-2015 is again associated with the tidal stream. However, Petrov et al. (2015) found changes in certain wind features and that the absorber covered only the star and its immediate surroundings. They proposed that the occultation was related to an interaction between the stellar wind and the inner disk, which is supported by enhanced $M$ - and $L$-band fluxes (Shenavrin et al. 2015). In this case, there might be similarities with UXor events observed in more massive stars (e.g., Grinin et al. 1998). No substantial increase in column density of cold gas towards the dimmed star was found (Petrov et al. 2015).

RW Aur has also been observed by Chandra with ACIS-S for $54 \mathrm{ks}$ during the bright state (January 2013; Skinner \& Güdel 2014). The A-B pair was resolved and the data revealed that the secondary is more X-ray luminous than the primary and that it is moderately variable (factor of 1.5 within $60 \mathrm{ks}$ ).

$\mathrm{X}$-ray absorption is essentially independent of ionization, because only the inner atomic shells contribute to X-ray absorption. Typically, the ratio between X-ray absorption expressed as equivalent neutral hydrogen column density $\left(N_{\mathrm{H}}\right)$ and optical extinction $\left(A_{V}\right)$ is used as a measure of the absorber's gas-to-dust 
Table 1. Overview of the analyzed observations.

\begin{tabular}{lccr}
\hline \hline Observatory & ObsID & Detector & Date(s) \\
\hline IRTF & 2015A023 & SpeX & 20-Mar.-2015 \\
Chandra & 17644 & ACIS-S VFAINT & 16-Apr.-2015 \\
UKIRT & U/15A/D01 & UFTI & 18-Apr.-2015 \\
OLT & - & - & 09-20-Apr.-2015 \\
AAVSO & - & - & various \\
\hline
\end{tabular}

ratio. As RW Aur is already well characterized during the bright state, we use the increase in X-ray absorption to derive the gas and small grain content of the extra absorber, where "small" means that the grains are not opaque in X-rays (a few $\mu \mathrm{m}$ ) .

We present new X-ray and optical/near-IR (NIR) data obtained during the dim state in Sect. 2, derive the corresponding column densities in Sect. 3, and compare both measurements in Sect. 4 to investigate the properties of the extra absorber and the nature of the dimming event.

\section{Observations and data analysis}

The new X-ray, optical, and NIR observations obtained to characterize the dim state are summarized in Table 1.

\subsection{Chandra observation and data processing}

The new X-ray observation (exposure time: $35 \mathrm{ks}$ ) was performed with the same setup as the 2013 observation of RW Aur during the bright state, i.e., $1 / 8$ subarray readout was used to minimize the pileup of the X-ray bright B component (see Skinner \& Güdel 2014). Data reduction was performed using ciao version 4.6 (Fruscione et al. 2006). Unless otherwise noted, the $0.3-10.0 \mathrm{keV}$ energy range was used.

Figure 1 shows the X-ray images as well as source and background extraction regions. The location of the 2013 jet emission (green arrow in Fig. 1) is outside our source extraction region and so does not affect our analysis. The source region (radius: 0.'54, PSF fraction: $75 \%$ ) contains 51 photons and we estimate that PSF spillover by RW Aur B contributes 21.4 photons. We checked that the exact parameters of the regions do not significantly influence the spectral data.

We modeled the stellar X-ray emission in xspec using photoelectric absorption (phabs) and vapec models with plasma abundances from Skinner \& Güdel (2014) for consistency. The equivalent hydrogen column density depends on the assumed absorber abundances; we used Anders \& Grevesse (1989). Lower metallicities as suggested by Asplund et al. (2009) increase $N_{\mathrm{H}}$ by about a factor of 1.5. To provide a robust estimate of the absorbing column density given the limited number of photons in 2015, we modeled the 2015 emission as a scaled version of the 2013 two component model plus extra absorption, i.e., we fixed the emission measure ratio ( $\left.E M_{\text {hot }} / E M_{\text {cool }}\right)$ to values that accurately describe the 2013 Chandra data. We also investigated several other models (e.g., free $E M_{\text {hot }} / E M_{\text {cool }}$ ratio, one component thermal plasma with free temperature) and find that the results are generally consistent with this approach.

Here, we are exclusively interested in the evolution of RW Aur A and note that the average X-ray flux of the B component increased by a factor 1.4 at similar spectral properties (unabsorbed $L_{\mathrm{X}}=1.9 \times 10^{30} \mathrm{erg} \mathrm{s}^{-1}$ ) with respect to 2013 and that its count rate smoothly decreases by a factor of 1.3 during the exposure. X-ray emission from the RW Aur A jet as described by Skinner \& Güdel (2014) is not significantly detected.

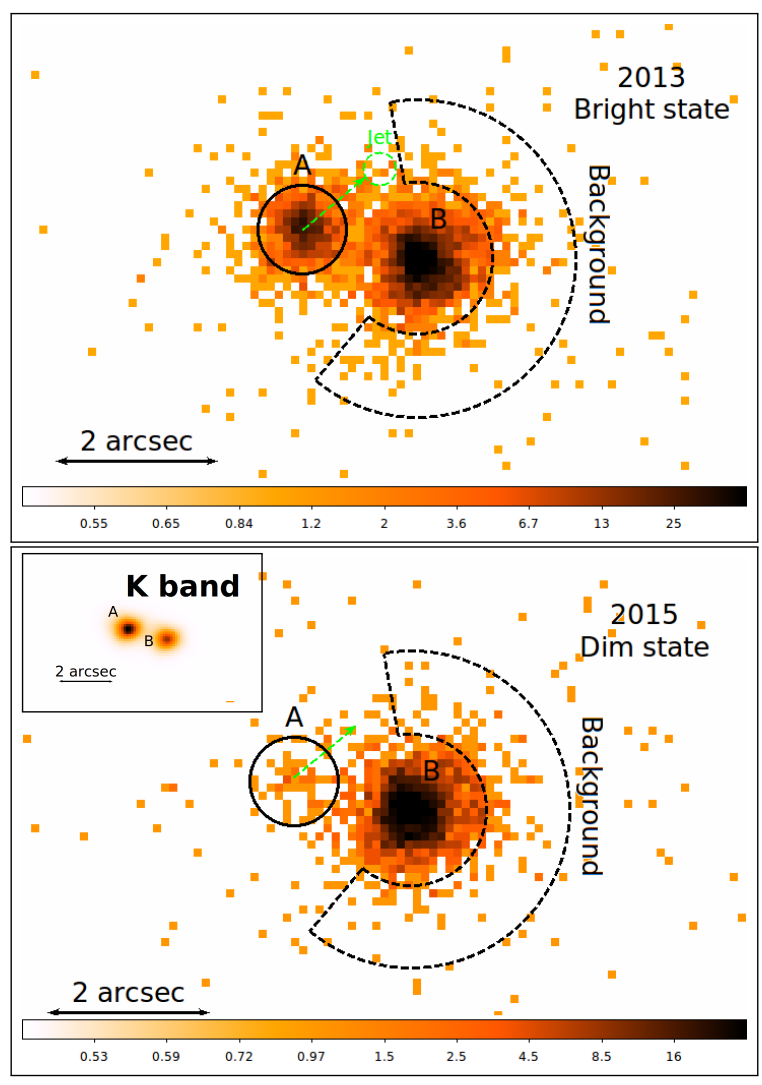

Fig. 1. X-ray images of RW Aur taken during the bright state in 2013 (top) and during the dim state in 2015 (bottom); the color bars indicate counts per pixel. The scaling reflects the difference in exposure time, i.e., similar count rates have similar colors. The inset shows the same sky area in the $K$-band obtained two days after the X-ray observation.

\subsection{Ground-based data}

Optical data in Johnson-Cousins filters were obtained with the Oskar-Lühning-Teleskop (OLT) at the Hamburger Sternwarte. Data reduction followed standard procedures. We also report AAVSO $^{1} V$ magnitudes that densely sample the optical brightness evolution of the system. When near-simultaneous OLT data are available, both datasets are compatible. Near-IR data were provided by IRTF and UKIRT. They were reduced using IRAF and Starlink ${ }^{2}$, respectively. The inset in Fig. 1 shows a UKIRT $K$-band image as an example of our NIR photometry. Absolute photometry was obtained by calibration against the 2MASS magnitudes of a check field and the standard star FS 12 (Hawarden et al. 2001) for the data from 20 March 2015 and 18 April 2015, respectively.

To estimate the brightness drop of RW Aur A, we compare the dim state values to its average bright state values, which we estimate from the ROTOR data (Grankin et al. 2007) transforming their Johnson $R$ to Cousin $R$ using the Bessell (1979) relations. For the NIR, we use the 2MASS magnitudes (Skrutskie et al. 2006) as the bright state reference. The contribution of the B component to the optical/NIR flux is below $10 \%$ during the bright state, but important during the dim state. For spatially unresolved observations, we subtract the average brightness of $\mathrm{B}$ calculated from resolved data to obtain the magnitude of A (see Table A.1).

\footnotetext{
1 Observations from the AAVSO International Database, http: //www . aavso.org

2 http://starlink.eao.hawaii.edu/starlink
} 


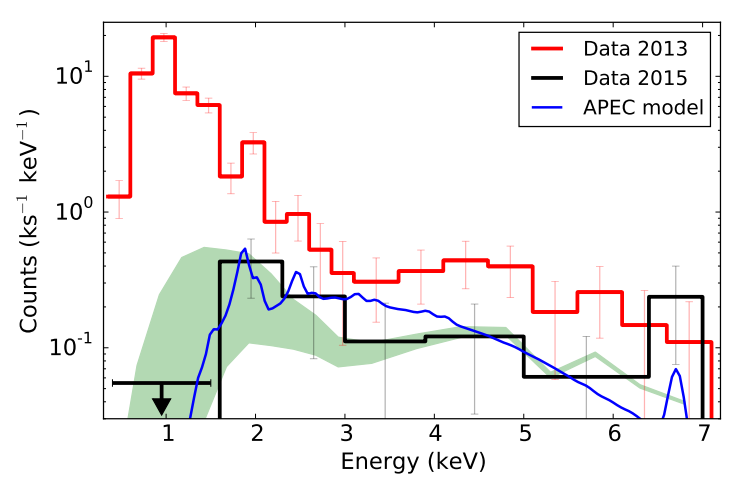

Fig. 2. X-ray spectra of RW Aur A with models. The APEC model uses the parameters provided in Table 2. The green shaded area shows the 2013 data scaled to match at $E_{\text {phot }}>3 \mathrm{keV}$ including absorption; lower and upper bounds pertain to $N_{\mathrm{H}}^{\text {extra }}=10^{22} \mathrm{~cm}^{-2}$ and $4 \times 10^{22} \mathrm{~cm}^{-2}$, respectively. Data was binned for display purposes.

Table 2. X-ray properties of RW Aur A.

\begin{tabular}{lccc}
\hline \hline Parameter & Value 2013 & Value 2015 & Unit \\
\hline Rate $(0.3-2.0 \mathrm{keV})$ & $12.3 \pm 0.4$ & $0.1 \pm 0.1$ & $\mathrm{cts} \mathrm{ks}^{-1}$ \\
Rate $(2.0-10.0 \mathrm{keV})$ & $2.0 \pm 0.2$ & $0.7 \pm 0.1$ & $\mathrm{cts} \mathrm{ks}^{-1}$ \\
$F_{\mathrm{X}}(0.3-10 \mathrm{keV}):$ & $1.67 \times 10^{-13}$ & $4.7 \times 10^{-14}$ & $\mathrm{erg} \mathrm{cm}^{-2} \mathrm{~s}^{-1}$ \\
$F_{\mathrm{X}}(2.0-10 \mathrm{keV}):$ & $6.1 \times 10^{-14}$ & $2.8 \times 10^{-14}$ & $\mathrm{erg} \mathrm{cm}^{-2} \mathrm{~s}^{-1}$ \\
$N_{\mathrm{H}}$ & $0.08_{-0.06}^{+0.06}$ & $2.3_{-1.0}^{+1.6}$ & $10^{22} \mathrm{~cm}^{-2}$ \\
$k T_{\text {cool }}$ & \multicolumn{2}{c}{$0.63_{-0.07}^{+0.12}$} & $\mathrm{keV}$ \\
$k T_{\text {hot }} 20.0$ & $\mathrm{keV}$ \\
$E M_{\text {hot }} / E M_{\text {cool }}$ & \multicolumn{2}{c}{$0.84 \pm 0.30$} & \\
C-statistic/bins & $180.30 / 221$ & $61.45 / 65$ & \\
\hline
\end{tabular}

Notes. Model with identical $E M_{\text {hot }} / E M_{\text {cool }}$ ratio in both epochs and $k T_{\text {hot }}$ fixed to $20 \mathrm{keV}$. Errors indicate $90 \%$ confidence ranges. Bin widths are 0.05 and $0.15 \mathrm{keV}$ for 2013 and 2015, respectively.

\section{Results}

In the following, we show that the gas and dust absorption strongly increased between the bright and dim states.

\subsection{X-ray data}

Figure 2 compares the X-ray spectra during the bright and dim states. The observed (absorbed) flux of the A component decreased by almost a factor of 20 (see Table 2) mainly owing to the decrease at soft photon energies. A significant source signal is only recorded above $1.9 \mathrm{keV}$. The number of photons at lower photon energies is compatible with a background fluctuation at the $90 \%$ confidence range. In addition, the observed flux above $2 \mathrm{keV}$ is reduced by about a factor of three.

The absorbing column density is rather well constrained because a hot component is needed to describe the high-energy tail of the observed spectrum $\left(E_{\text {phot }}>2 \mathrm{keV}\right)$ and emission from this component extends well into the soft part of the spectrum and drives the required absorbing column density. The fit results, summarized in Table 2, show that the absorbing column density increased by almost a factor of 30 to $N_{\mathrm{H}} \approx 2 \times 10^{22} \mathrm{~cm}^{-2}$ in 2015. The temperature of the hot component was fixed as its precise value is unconstrained (already noted by Skinner \& Güdel 2014). However, this does not affect the resulting $N_{\mathrm{H}}$ significantly. The best fit column density corresponds to $5 \times 10^{-2} \mathrm{~g} \mathrm{~cm}^{-2}$ for a mean molecular weight of $\mu=1.4$ and to $A_{V}=13 \mathrm{mag}$ for ISM-like absorption $\left(N_{\mathrm{H}}=1.8 \times 10^{21} \mathrm{~cm}^{-2} A_{V}^{-1}\right.$, Predehl \& Schmitt 1995; Vuong et al. 2003).

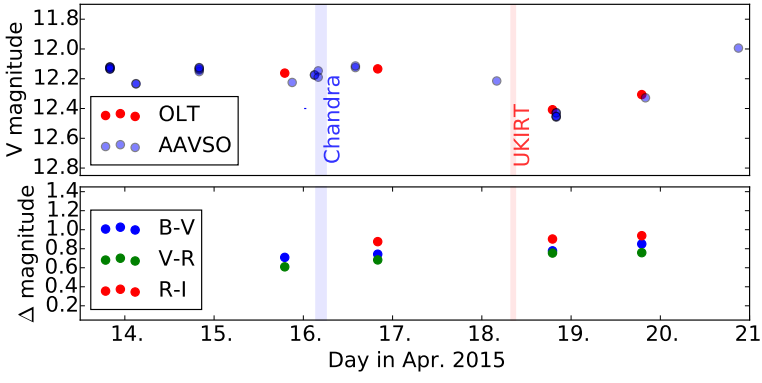

Fig. 3. Optical light curve of RW Aur around the X-ray observation (spatially unresolved).

\subsection{Optical and NIR data}

Figure 3 shows the $V$-band light curve as well as the color evolution around the Chandra observation. Only minor variability is seen around the X-ray observation (standard deviation in $V$ is $0.14 \mathrm{mag}$ on a ten-day time scale). Attributing this variability to the primary suggests intrinsic changes by only $0.2 \mathrm{mag}$ (color changes are $<0.1 \mathrm{mag}$ ), i.e., the optical properties are very similar during the Chandra and UKIRT observations. This strongly suggests that the UKIRT data closely approximates the conditions during the X-ray observation. Compared to variability during the bright state, these differences are small so that we regard our X-ray, optical, and NIR datasets as effectively simultaneous.

Figure 4 shows the drop in brightness as a function of wavelength with respect to the mean bright state. The drop at optical $(\triangle \overline{B V R} \sim 2.2)$ and $\operatorname{NIR}(\Delta \overline{J H K} \sim 1.9)$ wavelengths is almost identical, especially when considering that the bright state's reference magnitudes are somewhat uncertain owing to intrinsic variability. Therefore, ISM-like absorption, or minor variations thereof, are incompatible with the data (see blue and red curves in Fig. 4 top). Scattering of stellar photons in the circumstellar environment might contribute to the observed optical flux, which would erroneously indicate only little optical extinction possibly explaining why optical and NIR fluxes show a similar drop. However, the NIR is essentially unaffected by scattering because of its strong wavelength dependence $\left(\sigma_{\text {scat }} \sim \lambda^{-4}\right)$. Thus, substantial dust extinction should offset RW Aur from the CTTS locus (Meyer et al. 1997) along the reddening vector while the RW Aur colors are below the CTTS locus (Fig. 4 bottom), i.e., the NIR colors indicate no significant reddening. Without scattering, the evolution of the RW Aur A optical/NIR magnitudes are almost wavelength independent, i.e., gray. This requires an absorber consisting mainly of large grains and only a small amount of small grains, which is compatible with the findings by Antipin et al. (2015) from their optical data.

Gray absorption up to NIR wavelengths requires dust composed of grains with sizes $\gtrsim 1 \mu \mathrm{m}$. The ratio between extinction and mass decreases with increasing grain size. Therefore, assuming grains of $1 \mu \mathrm{m}$ in size provides a lower limit on the dust mass. These grains have absorption efficiencies around 2.5 (see, e.g., Draine \& Lee 1984) so that the mass attenuation coefficient is $10^{4} \mathrm{~cm}^{2} \mathrm{~g}^{-1}$ for dust densities of $\rho \approx 2 \mathrm{~g} \mathrm{~cm}^{-3}$ (e.g., carbonaceous grains, which dominate ISM dust at these sizes). The required dust mass column density is about $2 \times 10^{-4} \mathrm{~g} \mathrm{~cm}^{-2}$ for gray extinction of $2 \mathrm{mag}$.

\section{Interpretation and conclusions}

First, we assume the simplest scenario that reasonably explains our data: one single absorber, which contains the gas that causes the X-ray absorption and the large dust grains that cause the gray 

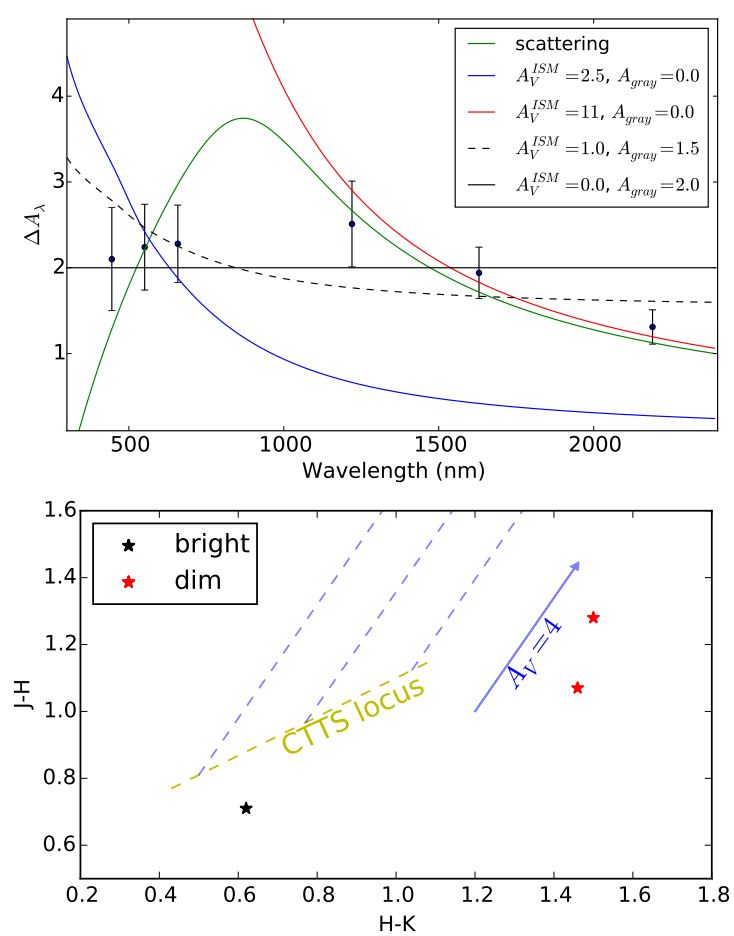

Fig. 4. Top: extinction by the extra absorber with different extinction curves. ISM-like models have $A_{\text {gray }}=0$ and $R_{V}=3.1$. The scattering model assumes ISM-like extinction with $A_{V}=10 \mathrm{mag}$, a scattering efficiency of $13 \%$ in $V$, and no additional extinction along the scattering path. Photometric bands are shown at their effective wavelengths. Bottom: NIR color-color diagram.

optical/NIR extinction. The requirement of predominately large grains is compatible with the $10 / 20 \mu$ m features seen in some IR spectra of CTTSs, which also suggest large grains in the $\mu \mathrm{m}$ size range (e.g., Oliveira et al. 2010). Combining X-ray and dust absorption, we derive an upper limit on the gas-to-dust ratio for this absorber of 250:1 with about a factor of two uncertainty (a factor of 1.7 in X-ray column density and we assume a factor of 1.5 in dust mass density $\rho$ ). Thus, one absorber with an ISM-like gas-to-dust ratio can explain the X-ray and optical data for processed dust, i.e., for dust with sizes between about 1 and $10 \mu \mathrm{m}$. This suggests that grains grow from their ISM distribution with a peak in the few $0.1 \mu \mathrm{m}$ range to sizes that are about ten times larger without strongly altering the gas-to-dust ratio. Without a substantial increase in low-ionization $\mathrm{Na}$ and $\mathrm{K} \mathrm{I}$ absorption as suggested by Petrov et al. (2015), the strong increase in X-ray absorption indicates that the absorbing material is hot. This suggests that the absorbing material is located close to the star and might also be responsible for the enhanced $L$ - and $M$-band fluxes measured by Shenavrin et al. (2015), e.g., the absorber might be a disk warp or an inner, dust-loaded wind as speculated by Petrov et al. (2015).

Second, we note that X-ray and dust extinction are not necessarily cospatial. The gas absorption might be caused by dustdepleted accretion streams or winds launched from the disk rim as in AA Tau where the innermost region $(\sim 0.1 \mathrm{au})$ is strongly gas-enhanced $\left(\Delta N_{\mathrm{H}} \approx 10^{22} \mathrm{~cm}^{-2}\right.$; see Schmitt \& Robrade 2007; Grosso et al. 2007). In addition to this gas absorbing component, an opaque structure that partly occults RW Aur A causes the gray extinction extending from the NIR to the highest energies in our X-ray spectrum. This opaque structure might be also located in the inner disk $(0.1 \mathrm{au})$ as the gas is, but could also be located slightly farther out at a few au - like the absorber that causes the long-lasting dimming of AA Tau (Bouvier et al. 2013; Schneider et al. 2015) - or could even be part of the tidal stream. Depending on the actual location of the absorber, this likely requires a tilt between the inner and the outer disk resolved by the $\mathrm{CO}$ observations given that the upper limit on the inclination of the RW Aur A disk is $i<60^{\circ}$, while that of AA Tau is $i \approx 75^{\circ}$. Such a scenario appears attractive, because it releases the constraint that the grain size distribution is missing grains smaller than $\lesssim 1 \mu \mathrm{m}$ and provides a uniform explanation for AA Tau-like CTTS and RW Aur A.

Acknowledgements. We thank B. Wilkes for granting the Chandra DDT observation and the referee, Prof. G. Gahm, for the careful and constructive report. P.C.S. and C.F.M. gratefully acknowledge an ESA Research Fellowship, H.M.G. is supported by NASA-HST-GO-12315.01, S.J.W. NASA contract NAS8-03060 (Chandra), and S.F. by an STFC/Isaac Newton Trust studentship. The results reported are based on observations made by the Chandra X-ray Observatory and by the United Kingdom Infrared Telescope (UKIRT) supported by NASA and operated under an agreement among the University of Hawaii, the University of Arizona, and Lockheed Martin Advanced Technology Center; operations are enabled through the cooperation of the Joint Astronomy Centre of the Science and Technology Facilities Council of the UK and by UKIRT. Some photometry was obtained at the Infrared Telescope Facility (IRTF), which is operated by the University of Hawaii under contract NNH14CK55B with the National Aeronautics and Space Administration (NASA).

\section{References}

Anders, E., \& Grevesse, N. 1989, Geochim. Cosmochim. Acta, 53, 197 Antipin, S., Belinski, A., Cherepashchuk, A., et al. 2015, Information Bulletin on Variable Stars, 6126, 1

Asplund, M., Grevesse, N., Sauval, A. J., \& Scott, P. 2009, ARA\&A, 47, 481

Bessell, M. S. 1979, PASP, 91, 589

Bouvier, J., Alencar, S. H. P., Harries, T. J., Johns-Krull, C. M., \& Romanova, M. M. 2007, Protostars and Planets V, 479

Bouvier, J., Grankin, K., Ellerbroek, L. E., Bouy, H., \& Barrado, D. 2013, A\&A, 557, A77

Cabrit, S., Pety, J., Pesenti, N., \& Dougados, C. 2006, A\&A, 452, 897

Cutri, R. M., Skrutskie, M. F., van Dyk, S., et al. 2003, VizieR Online Data Catalog: II/246

Dai, F., Facchini, S., Clarke, C. J., \& Haworth, T. J. 2015, MNRAS, 449, 1996

Draine, B. T., \& Lee, H. M. 1984, ApJ, 285, 89

Fruscione, A., McDowell, J. C., Allen, G. E., et al. 2006, in SPIE Conf. Ser., 6270,1

Grankin, K. N., Melnikov, S. Y., Bouvier, J., Herbst, W., \& Shevchenko, V. S. 2007, A\&A, 461, 183

Grinin, V. P., Rostopchina, A. N., \& Shakhovskoi, D. N. 1998, Astron. Lett., 24 802

Grosso, N., Bouvier, J., Montmerle, T., et al. 2007, A\&A, 475, 607

Hawarden, T. G., Leggett, S. K., Letawsky, M. B., Ballantyne, D. R., \& Casali, M. M. 2001, MNRAS, 325, 563

Meyer, M. R., Calvet, N., \& Hillenbrand, L. A. 1997, AJ, 114, 288

Oliveira, I., Pontoppidan, K. M., Merín, B., et al. 2010, ApJ, 714, 778

Petrov, P. P., \& Kozack, B. S. 2007, Astron. Rep., 51, 500

Petrov, P. P., Gahm, G. F., Gameiro, J. F., et al. 2001a, A\&A, 369, 993

Petrov, P. P., Pelt, J., \& Tuominen, I. 2001b, A\&A, 375, 977

Petrov, P. P., Gahm, G. F., Djupvik, A. A., et al. 2015, A\&A, 577, A73

Predehl, P., \& Schmitt, J. H. M. M. 1995, A\&A, 293, 889

Rodriguez, J. E., Pepper, J., Stassun, K. G., et al. 2013, AJ, 146, 112

Schmitt, J. H. M. M., \& Robrade, J. 2007, A\&A, 462, L41

Schneider, P. C., France, K., Günther, H. M., et al. 2015, A\&A, 584, A51

Shenavrin, V. I., Petrov, P. P., \& Grankin, K. N. 2015, Information Bulletin on Variable Stars, 6143, 1

Skinner, S. L., \& Güdel, M. 2014, ApJ, 788, 101

Skrutskie, M. F., Cutri, R. M., Stiening, R., et al. 2006, AJ, 131, 1163

Vuong, M. H., Montmerle, T., Grosso, N., et al. 2003, A\&A, 408, 581

White, R. J., \& Ghez, A. M. 2001, ApJ, 556, 265

Williams, J. P., \& Cieza, L. A. 2011, ARA\&A, 49, 67

Page 5 is available in the electronic edition of the journal at http://www . aanda.org 


\section{Appendix A: RW Aur photometry}

Table A.1 lists the optical/NIR magnitudes used in this study.

Table A.1. Optical and NIR magnitudes ordered by observing date.

\begin{tabular}{|c|c|c|c|c|c|c|c|}
\hline Date & Band & Resolved & System & RW Aur A & RW Aur B & Obs. & Ref. $^{a}$ \\
\hline 06-Sep.-1986 & $B$ & $\mathrm{n}$ & $11.18 \pm 0.48$ & $11.23 \pm 0.56$ & mean & ROTOR & (1) \\
\hline \multirow{3}{*}{ - 11-Oct.-2013 } & V & $\mathrm{n}$ & $10.45 \pm 0.40$ & $10.54 \pm 0.50$ & mean & & \\
\hline & $R_{\mathrm{J}}$ & $\mathrm{n}$ & $9.59 \pm 0.31$ & & & & \\
\hline & $R_{\mathrm{C}}$ & $\mathrm{n}$ & $9.85 \pm 0.31$ & $9.97 \pm 0.43$ & mean & & \\
\hline \multirow{3}{*}{ 09-Nov.-1994 } & $B$ & $\mathrm{y}$ & 10.99 & $11.02 \pm 0.02$ & $14.89 \pm 0.10$ & HST & (2) \\
\hline & V & $\mathrm{y}$ & 10.45 & $10.51 \pm 0.02$ & $13.63 \pm 0.05$ & & \\
\hline & $R$ & $\mathrm{y}$ & 9.86 & $9.94 \pm 0.03$ & $12.69 \pm 0.03$ & & \\
\hline 06-Dec.-1996 & K & & 6.83 & $7.06 \pm 0.17$ & $8.64 \pm 0.36$ & & \\
\hline \multirow[t]{3}{*}{ 27-Nov.-1999 } & $J$ & $\mathrm{n}$ & $8.38 \pm 0.02$ & 8.64 & mean & 2MASS & (3) \\
\hline & $H$ & $\mathrm{n}$ & $7.62 \pm 0.04$ & 7.93 & mean & & \\
\hline & $K_{\mathrm{S}}$ & $\mathrm{n}$ & $7.02 \pm 0.02$ & 7.31 & mean & & \\
\hline \multirow[t]{3}{*}{ 13/14-Dec.-2014 } & $B$ & $\mathrm{y}$ & 13.62 & $14.50 \pm 0.06$ & $14.26 \pm 0.05$ & Caucasus & (4) \\
\hline & $V$ & $\mathrm{y}$ & 12.52 & $13.80 \pm 0.05$ & $12.92 \pm 0.03$ & & \\
\hline & $R_{\mathrm{c}}$ & $\mathrm{y}$ & 11.66 & $13.18 \pm 0.07$ & $11.97 \pm 0.07$ & & \\
\hline \multirow{3}{*}{ 20-Mar.-2015 } & $J$ & $\mathrm{y}$ & 9.43 & $10.65 \pm 0.05$ & $9.85 \pm 0.04$ & UKIRT & (5) \\
\hline & $H$ & $\mathrm{y}$ & 8.53 & $9.58 \pm 0.04$ & $9.05 \pm 0.04$ & & \\
\hline & $K$ & $\mathrm{y}$ & 7.57 & $8.12 \pm 0.03$ & $8.57 \pm 0.03$ & & \\
\hline 06-Apr.-2015 ${ }^{b}$ & V & $\mathrm{n}$ & $12.17 \pm 0.14$ & 12.70 & mean & AAVSO & (5) \\
\hline - 26-Apr.-2015 & $R$ & $\mathrm{n}$ & $11.47 \pm 0.08$ & 12.15 & mean & & \\
\hline 09-Apr.-2015 & $B$ & $\mathrm{n}$ & $13.02 \pm 0.15$ & 13.34 & mean & OLT & (5) \\
\hline \multirow[t]{2}{*}{ - 19-Apr.-2015 } & V & $\mathrm{n}$ & $12.25 \pm 0.11$ & 12.84 & mean & & \\
\hline & $R$ & $\mathrm{n}$ & $11.55 \pm 0.07$ & 12.31 & mean & & \\
\hline \multirow[t]{3}{*}{ 18-Apr.-2015 } & $J$ & $\mathrm{y}$ & 9.90 & $11.15 \pm 0.05$ & $10.32 \pm 0.04$ & UKIRT & (5) \\
\hline & $H$ & $\mathrm{y}$ & 8.76 & $9.87 \pm 0.03$ & $9.24 \pm 0.04$ & & \\
\hline & $K$ & $\mathrm{y}$ & 7.74 & $8.37 \pm 0.04$ & $8.63 \pm 0.03$ & & \\
\hline
\end{tabular}

Notes. For unresolved observations, the magnitudes of the A component were calculated by subtracting the average brightness of the B component estimated from the resolved observations with the error assumed as the standard deviation $\left(B_{B}=14.5 \pm 0.3, V_{B}=13.2 \pm 0.3, R_{B}=12.3 \pm 0.3\right.$, $\left.J_{B}=10.06 \pm 0.3, H_{B}=9.14 \pm 0.1, K=8.61 \pm 0.04\right)$. For resolved data, the system's magnitudes are also provided by summing both components.

References. ${ }^{(a)}$ References as follows: (1) Grankin et al. (2007); (2) White \& Ghez (2001); (3) Cutri et al. (2003); (4) Antipin et al. (2015); (5) this work. ${ }^{(b)}$ Values pertain to mean and standard deviation during this time. 